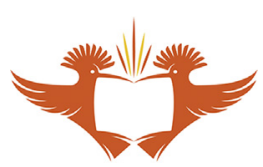

$\frac{\text { UNIVERSITY }}{\text { JOHANNESBURG }}$

\title{
Editorial Article
}

\section{Editorial comments on 2016 volume of HSAG}

Authors from 16 South African universities, 6 international universities and 6 other organisations published in Health SAGesondheid. The South African universities that published were: University of Cape Town, Cape Peninsula University of Technology, Durban University of Technology, University of the Free State, University of Johannesburg, University of KwaZuluNatal, University of Limpopo, Nelson Mandela Metropolitan University, North West University, Rhodes University, Sefako Makgatho Health Sciences University, Tshwane University of Technology, University of South Africa, Western Cape University and the University of Witwatersrand. The international universities that also published in HSAG were: Buckinghamshire New University, Mahidol University, Vrije Universiteit Amsterdam, Virginia Commonwealth University, The University of Western Australia.

University of Malawi, Other organisations that were included are: Earth Science Institute, Australia; Human Science Research Council, SA Medical Research Council, South African Non-academic institutions, Extreme Wellness and Somar Solutions,; Pharmasoft research, Wilkoppies informal, SA and SA database for functional research.

The different disciplines represented by our authors were: Anaesthesiology, Biostatistics, Clinical Care Sciences, Ear Sciences, Education, Emergency Medical Care, Health Development, Health and Rehabilitation, Health Behaviour, Policy, Health Studies, Infectious Diseases, Radiographics, Medical Sciences, Nursing Science, Nutrition and Dietetics, Occupational Therapy, Obstetrics, Gynaecology and Pharmaceutical Sciences, Psychiatric Nursing, Public Health Care and Speech-Language Pathology and Audiology. 11 articles identified themes from the 47 published articles are: HIV, education, destructive behaviour, medical imaging and radiation, cardiovascular, pharmacology, self-care, emergency medical care, blood-work, mother and child health and ethical transgressions in practice.

16 articles focused on issues pertaining to HIV prevention and care of persons living with HIV (PLWH). The articles addressing prevention of HIV were about reproductive services, mother to child transmission, transmission to health professionals, the use of female condoms and risky sexual behaviour. Articles on caring addressed testing for HIV,

Peer review under responsibility of Johannesburg University. psychosocial wellbeing of nurses caring for PLWH, stigma reduction and tuberculosis care. 10 articles addressed educational issues. 7 studies addressed tertiary education in which the emerging themes in tertiary education were clinical judgement in nursing, caring of student nurses and patients, systematic review as research method and professional development of occupational health nurses. 3 articles addressed secondary school education. The secondary school articles focused on learners' health including hearing screening and physical activity.

Destructive behaviours reflected in 6 articles. Self-harm as demonstrated by young women living with borderline personality disorder, nyaope (also known as whoonga or wunga) is a highly addictive, dangerous and destructive street drug, unique to South Africa users, attitudes and observation of self-harm practices and suicide were addressed in 5 articles and violence against nurses in 1 article. Radiography as a theme was reflected in 4 articles with topics such as data security and breaches, Doppler screening in prediction of preeclampsia, selection criteria for students and critical ability of students. 3 articles focused on cardiovascular issues such as risk factors and knowledge of African men, cardiovascular workout and cardiovascular functioning and fitness index. 3 articles focused on pharmacology, Two articles addressed the possible cost saving of generic medication and 1 article focused on pharmacological vigilance of pharmacists.

3 articles were published with self-care as a theme. Selfcare referred to the Gamma nursing measure assessing patients' activity economic status to the independent application of self-care of older persons and living with breast cancer related lymphedema. 2 articles on blood work addressed appropriate blood product use in perioperative patients and nurse phlebotomy competencies. The theme emergency medical care was addressed in 2 articles on limiting interruptions to CPR and paramedics experiences of financial medicine practices. Lastly the theme of mothers and babies was reflected in two articles on mothers' perceptions of their premature infant roll-out of kangaroo mother care. Lastly ethics transgressions in health care practice were addressed in 1 article.

In conclusion it is evident that prevention and care of persons living with HIV, education in the health sciences, 
preventing destructive behaviour and issues in radiography seems to be themes of interest in the health sciences fraternity.

Marie Poggenpoel, RN, PhD, fANSA, Professor: Nursing, Editor-in-Chief Department of Nursing, University of Johannesburg, Sixth Floor, West Wing North, John Orr Building, 6104e, C/O Beit and Nind Street, Doornfontein, South Africa E-mail address: mariep@uj.ac.za
8 December 2016 Available online 9 March 2017 1025-9848/@ 2017 The Author. Publishing services by Elsevier B.V. on behalf of Johannesburg University. This is an open access article under the CC BY-NC-ND license (http://creativecommons. org/licenses/by-nc-nd/4.0/). 